Cómo citar este artículo en MLA: Ghisiglieri, Francisco y Andrea Gigena. "Familismo como posibilidad vincular en la subjetivación de jóvenes en condiciones de pobreza en Córdoba (Argentina)”. Escritos 28. 60 (2020): 93-108 doi: http://dx.doi.org/10.18566/escr.v28n60.a09

Fecha de recepción: 13.04 .2020

Fecha de aceptación: 10.06.2020

\title{
Familismo como posibilidad vincular en la subjetivación de jóvenes en condiciones de pobreza en Córdoba (Argentina)
}

\author{
Familism as Linking Possibility in the Subjectivation of Youngsters in Poverty \\ Condition in Cordoba (Argentina)
}

\author{
Francisco Ghisiglieri ${ }^{1}$ \\ Andrea Gigena ${ }^{2}$ ()
}

\section{RESUMEN}

El trabajo aborda los procesos de subjetivación de jóvenes en condiciones de pobreza de un barrio de la Ciudad de Córdoba (Argentina). Desde una perspectiva analítica foucaultiana, se analizaron los modos en que los sujetos se constituyen en un devenir histórico, a través de un entramado de prácticas de gobierno y resistencia. El trabajo, de alcance analítico-interpretativo, tiene como objetivo principal indagar cómo las y los jóvenes de este barrio construyen sus subjetividades en un contexto de exclusión social. En particular, se analiza en el caso estudiado la relevancia que adquieren los vínculos familiares y las implicancias que tiene para las mujeres este repliegue. El trabajo de campo se realizó entre los años 2013 y 2017. Se realizaron registros de observación y análisis de noticias periodísticas y comunicaciones gubernamentales. Se observa una forma de subjetivación en las y los jóvenes que refuerza la pertenencia familiar como un modo posible de sostener el lazo social frente a la fragmentación social y la estigmatización que atraviesan su vida. Este repliegue familista, a su vez, promueve un espacio en el que las mujeres resultan reducidas al rol de madres y/o cuidadoras y, desde ese lugar, sobrecargadas por las exigencias cotidianas. Estas características se dan en un contexto de políticas de Estado que consolidaron a la familia nuclear como eje de sus intervenciones y, con las reorientaciones que implicó el neoliberalismo, como

1 Licenciado en Psicología por la Universidad Católica de Córdoba, Argentina. Miembro de los grupos de investigación: "El rol de la escuela en la formación de jóvenes resilientes: un estudio comparativo de bullying y ciberbullying entre escuelas públicas y privadas" - Instituto de Investigaciones Psicológicas (Conicet-Universidad Nacional de Córdoba, Argentina); y "Los vínculos grupales en la formación universitaria: dispositivos de reflexión, promoción de salud mental y ciudadanía responsable" (Universidad Católica de Córdoba). Correo electrónico: franciscoghisiglieri@ gmail.com.

2 Doctora en Ciencias Sociales por la Universidad de Buenos Aires, Argentina. Investigadora, Conicet - Universidad Católica de Córdoba, Argentina. Correo electrónico: aigigena@conicet.gov.ar. 
proveedoras exclusivas de cuidados. Para concluir, se abren interrogantes sobre las posibilidades de este familismo de convertirse en un soporte de procesos comunitarios más amplios.

Palabras clave: Familismo; Procesos de subjetivación; Jóvenes; Pobreza; Género; Vínculos familiares.

\begin{abstract}
The article considers the processes of subjectivation of youngsters in poverty condition of a neighborhood of Cordoba (Argentina). From a Foucauldian analytic standpoint, it analyzes the ways in which subjects constitute themselves through a historical development and within a framework of practices of government and resistance. Therefore, the purpose of the article is to understand how the youngsters of the neighborhood built their subjectivities within a context of social exclusion. Particularly, it analyzes the relevance of kinship and the consequences for women. The fieldwork was undertaken between 2013 and 2017 by means of observation record and analysis of newspapers and government communications. The way of subjectivation that was observed in the youngsters reinforces kinship as a possible way to maintain social bonds despite the social fragmentation and stigmatization that they experience throughout their lives. In turn, this familist retreat fosters a space in which women are reduced to the role of mothers or caretakers, and because of that, are overwhelmed by everyday demands. These features occurred within a context of State policies that made nuclear family their aim of intervention and, also because of neoliberalism, the only providers of care. It concludes with some questions regarding the possibilities of such familism to become the foundation for broader community processes.
\end{abstract}

Keywords: Familism; Processes of Subjectivation; Youngster; Poverty; Gender; Kinship.

\title{
Introducción
}

$\mathrm{E}$ ste artículo presenta resultados de una investigación llevada adelante en Barranca Yaco, un barrio en condiciones de pobreza de la Ciudad de Córdoba (Argentina). El objetivo general fue analizar los procesos de subjetivación de los y las jóvenes que habitan este sector de la Ciudad. El marco general de la investigación fue la tesis para optar por el grado de Doctor "Procesos de subjetivación de jóvenes en condiciones de pobreza en un barrio de la Ciudad de Córdoba (Argentina)", de la Facultad de Psicología de la Universidad Nacional de Córdoba, y el trabajo pudo ser realizado por una beca cofinanciada Conicet - Universidad Católica de Córdoba (2014-2019).

Para este análisis se tomaron herramientas conceptuales foucaultianas (Foucault, El sujeto; Verdade; Arqueología; Defender; Seguridad; Nacimiento de la biopolítica; Vigilar y Castigar; Historia de la sexualidad) referidas a los procesos de subjetivación. Desde los desarrollos de este autor, se comprende que el sujeto se constituye en un devenir histórico, a través de un entramado de relaciones de poder. Prácticas tanto de gobierno, como de resistencia, condicionan las posibilidades de pensar, sentir y actuar en cada contexto particular. En este sentido, abordar la subjetivación desde esta perspectiva implica necesariamente un análisis situado.

En este caso, una de las primeras condiciones a la que es necesario referir es la exclusión social de los y las jóvenes que habitan condiciones de pobreza en estos territorios de Latinoamérica (Nateras, Juventudes; Nateras, Geografías; Di Leo y Camarotti). Un contexto de exclusión social atravesado por múltiples formas de violencia (Reguillo; Míguez; Auyero y Berti; Cardozo; Di Leo y Pinheiro) que se producen y reproducen, especialmente, a través de dos tecnologías de gobierno íntimamente relacionadas: la policial y la territorial. 
En nombre de políticas de seguridad y de prevención (Crisafulli), la institución policial se convierte en un órgano central en la administración y distribución de violencia (Bisig; Comisión Provincial de la Memoria de Córdoba), así como en la gestión de las oportunidades de circular en la Ciudad (Cardozo) de la población juvenil.

A la par, las intervenciones en el territorio han diseñado en Córdoba un sofisticado dispositivo de control de los cuerpos que tiene entre sus principales efectos la fragmentación socio-territorial y la estigmatización de la pobreza (Scribano y Boito; Núñez y Ciuffolini; Cervio, Del barrio social). Como sostiene Grinberg, más que ausencia de articulación, estos efectos pueden leerse como parte de una racionalidad de gobierno que encuentra en la fragmentación un modo de ramificarse, dentro de una biopolítica.

Aquí debemos destacar, por su relevancia para el caso analizado y para el tejido social de la Ciudad de Córdoba en su conjunto, el Programa "Nuevos Barrios: Mi Casa, Mi Vida". Fue decretado en el año 2002 por el Gobierno Provincial y contó con financiamiento del Banco Interamericano de Desarrollo (Cervio, Del Barrio social); el mismo, mediante una importante intervención pública, aseguró la erradicación casi definitiva de los barrios humildes de las zonas centrales y peri-centrales de la Ciudad. Sobre las implicancias de este Programa existe abundante literatura (Scribano y Boito; Núñez y Ciuffolini; Scarponetti y Ciuffolini) En términos generales, la misma sostiene una visión crítica hacia el accionar del Estado y señalan las consecuencias negativas que tuvo esta política social para los sujetos afectados en términos de desconocimiento de sus necesidades, de complicidad con intereses de grandes grupos económicos y de aumento de la segregación socio-territorial.

A continuación presentamos un hallazgo del análisis: una forma de subjetivación que opera desde un reforzamiento de lo familiar como espacio de pertenencia privilegiado. Frente a la fragmentación social y la estigmatización que atraviesa la vida de los y las jóvenes, se observa un repliegue a los vínculos familiares como un modo de sostener el lazo social.

A su vez, la relevancia de lo familiar nos llevó a considerar las implicancias que esto tiene para las mujeres. Desde esta perspectiva, se puso en evidencia que lo familiar, a la par de constituirse en una pertenencia desde la cual se sostienen subjetivaciones posibles, también es un espacio en el que las mujeres resultan reducidas al rol de madres y/o cuidadoras y, desde ese lugar, sobrecargadas por las exigencias cotidianas. La metodología utilizada fue de tipo cualitativo y se tomaron herramientas de la analítica foucaultiana del poder (Foucault, Defender; Nacimiento de la biopolítica; Vigilar y Castigar; Historia de la sexualidad). El trabajo de campo se realizó duante cinco años (2013-2017), con una inserción a terreno cuyo vínculo principal fue un proyecto socio-educativo ubicado en el barrio, llamado Proyecto Guadix: un espacio laico gestionado por la Institución Teresiana (una asociación internacional de laicos de la Iglesia Católica), en el que participan aproximadamente 60 niños, niñas y jóvenes de entre 6 y 18 años, quienes asisten regularmente al apoyo escolar y a otras actividades socio-educativas. También participan familiares y personas allegadas del barrio que asisten a reuniones, encuentros y eventos comunitarios ocasionales. Durante el período mencionado, uno de los integrantes del equipo de investigación participó activamente del proyecto y realizó talleres junto con los y las jóvenes (de memoria colectiva, de cartografía, taller de radio). En este marco, se realizaron registros de campo, entrevistas a jóvenes y adultas/os,

También se incorporó el análisis de fuentes secundarias (noticias periodísticas, documentos gubernamentales y de difusión de organizaciones sociales y políticas). Estas fuentes nos brindaron, por un lado, información para aproximarnos a la realidad social del lugar. La recolección de datos brindó 
información que pudimos utilizar para formular preguntas, así como facilitó la contextualización de los datos que los entrevistados aportaban. Para el análisis, permitió principalmente triangular la información producida desde el terreno. ${ }^{3}$.

\section{El repliegue a los vínculos familiares como posibilidad vincular}

Podemos decir que una de las principales características de las relaciones sociales de Barranca Yaco es la superposición de los lazos comunitarios a los familiares. Como señalaba Rosana -una profesional de la zona-: "El barrio está organizado por familias, acá está la familia Gutiérrez, y vive la madre o la abuela al medio, y después se van anexando los que van creciendo". Son las relaciones familiares, $-\mathrm{y}$, como veremos, las mujeres tienen un rol protagónico en ellas-, las que modulan y moldean las demás relaciones posibles. Esto afecta la organización espacial, así como las redes de intercambio y cuidado.

Romina (Mujer adulta)- Por eso es que yo les decía que acá están todos en familia. Está el tío, el primo, el amigo, el cuñado, están todos metidos acá.

Voluntaria- O sea que Barranca Yaco es una comunidad familiar.

Liliana (Mujer adulta)- Algo así.

Romina- Es una comunidad familiar, pero cada familia tira para sí misma ¿Entendés? (Entrevista, 1 de noviembre de 2017).

Nos parece importante discriminar, de acuerdo con lo observado en el análisis general de la investigación, que esta superposición entre lo comunitario y lo familiar tiene dos derivas posibles, dos formas de apropiarse subjetivamente de esta condición familista: cuando las luchas de lo familiar no coinciden con lo comunitario, cuando no se logra gestar un acuerdo, lo familiar intercepta lo comunitario, los subyuga y los diluye. Su saldo son familias que se desencuentran en búsquedas aisladas - "cada familia tira para sí misma”. En cambio, cuando estas luchas coinciden, una dimensión apuntala la otra y es posible una acción colectiva (como, por ejemplo, en este caso, acciones en pos de garantizar el derecho a la vivienda [Cervio, La Ciudad]).

Entonces, en primer lugar, encontramos que lo familiar deviene en una práctica de repliegue a configuraciones familiares que se definen con perímetros cerrados. Esta característica, por ejemplo, se podía percibir en las redes que se tejen en las salas del Proyecto Socio-educativo, las cuales, por lo general, tienen como basamento las relaciones de parentesco.

Francisco- ¿Por qué te cambias de grupo?

Ana (joven)- Para que estemos igual, sino allá somos más.

Coordinadora- No, si están bien en cantidades.

No dice nada.

F- ¿Por qué te cambiaste?

Ana- Porque acá está mi hermana (se ríe). No, mentira.

3 Cabe aclarar que todos los nombres y referencias que podrían revelar la identidad de los sujetos participantes de la investigación han sido modificados. No así, las referencias históricas, ya que constituyen información que puede resultar útil para futuras investigaciones. 
F- Bueno, te invito a que te integres con otros amigos, no sólo de tu familia.

Silencio un ratito. Me mira y dice:

Ana- Bueno, yo no acepto su invitación.

Nos reímos y ella sigue trabajando con su hermana (Registro de campo, 19 de mayo de 2016).

En los y las jóvenes, específicamente, lo familiar como condición de vinculación aparece matizado por las relaciones barriales (de vecindad -en los casos que no coinciden con las relaciones familiares- y las pertenencias a instituciones como el club del barrio). La incidencia de la pertenencia escolar como una pertenencia capaz de promover el encuentro y las relaciones de amistad es llamativamente baja. Como se puede percibir en el fragmento anterior, el grupo de pares habitualmente resulta superpuesto con las relaciones familiares y dificultando la producción de nuevas relaciones extra-familiares.

En el siguiente fragmento de registro de campo se pueden percibir varios de los elementos característicos de este primer modo de apropiarse subjetivamente del repliegue familiar.

Francisco- A ver, si ustedes quisieran conocer la historia de alguien, ¿qué le preguntarían?

Lara (joven)- ¿Cómo se llama?

F- Bueno, escriban, uno, cómo se llama.

Juan (Joven)- Dale, escribíme (a una compañera que está escribiendo por él).

F- ¿Qué otra pregunta?

Laura (joven)- ¿Cuántos años tiene?

María (joven)- Su edad.

F- Bien... acuérdense que queremos saber la historia de esa persona ¿no?

Coordinador - Perfecto, ¿qué más le podemos preguntar para conocer su historia?

L- ¿Con quién vive? ¿Si tiene padre?... ¿Muertos o vivos?

M- Si tiene hermanos.

L- ¿Tenés abuelita?

F- Acuérdense que es la historia lo que queremos conocer.

L- ¿Tenés Facebook?

Coordinador- Es un gran reservorio de historia ese.

L- Yo tengo un amigo, viste, que tiene una foto en el Facebook, que se... cómo se llama, ese que pasan en

la tele a la mañana...

Coordinador- Canal 12.

L- Sí, bueno ahí se fue. Juega al fútbol.

F- Qué hizo... ¿esa sería la pregunta?

L- $\mathrm{O}$ ¿a qué se dedicó?

Conversan sobre quién tiene Facebook entre ellos, algunos tienen, otros no.

El Coordinador propone preguntar dónde nació.

Circulan preguntas al respecto: “¿Dónde naciste?” “Dónde vivís?”

L- Yo tengo una para la nueve... ¿qué color de ojos tienes?

Francisco- Pero, ¿qué tiene que ver con la historia eso?

L- ¿Sos igual a algún pariente tuyo? (...) (Registro de campo, 28 de octubre de 2015).

Desde el punto de vista de este grupo de jóvenes, a la hora de conocer la historia de alguien emerge como primer dato relevante conocer el modo en que está compuesta su familia; familia que está pre-definida desde un modelo nuclear y heterosexual. 
Es interesante que sobre la madre no se realicen preguntas, a pesar de la presencia que posee en la vida cotidiana de este barrio. Esto se puede leer en clave de una invisibilización de su figura, sumado a lo "impensable" de su ausencia. En cambio, sí se pregunta por la "abuela", figura relevante como agente de cuidados y poder en estos entramados.

Sobre el padre no sólo se pregunta, sino que se vuelve necesario averiguar si se encuentra con vida. Esto se hace comprensible por la habitual ausencia masculina adulta en los escenarios familiares y comunitarios. Lo anterior tiene diversas explicaciones posibles: muchos trabajan en jornadas extendidas que los mantienen fuera de sus hogares gran parte del tiempo, otros están en prisión. En otras ocasiones, su ausencia se debe a que los padres abandonan a sus hijos/as al cuidado de sus madres, las que aun así siguen fuera del horizonte de las preguntas. También observamos la existencia de episodios de violencia de género que provocan la situación de que las mujeres necesiten/deban llevar adelante sola las crianza y cuidado de los hijos y las hijas.

En el marco de estas prácticas familistas de la apropiación de lo familiar, emergen con insistencia situaciones de sobrecarga de las mujeres, particularmente las madres, responsables de manera exclusiva de las tareas de cuidado de sus hijos/as, así como víctimas, como ya mencionamos, de múltiples y variados episodios de violencia de género.

Rosana (una profesional de la zona)- Un tiempo hicieron un café con la psicóloga, trabajaban violencia de género. Ella estaba contándoles que era un problema que les pasa a todas, hasta a las mujeres más ricas, cuando María dice: “Uh, si les pasa a ellas... ¿qué no nos va a pasar a nosotras? Si no somos nada...” (Registro de campo, 16 de agosto de 2017).

La violencia de género se articula aquí con las demás desigualdades existentes y los procesos de objetivación destituyente $e^{4}$, produciendo este tipo de auto-percepciones-"si no somos nada"- que refuerzan y naturalizan lugares de sometimiento. Lo familiar como familismo se articula muchas veces con diversas modalidades de violencia de parte de los hombres hacia las mujeres: abandonos, extorsiones, golpizas, engaños; así como toda una gama de micro-machismos, que producen y reproducen las relaciones desiguales entre los géneros.

En resumen, esta primera apropiación subjetiva familista de lo familiar se caracteriza por poseer una frontera que encierra y bloquea las posibilidades de intercambio y articulación con lo comunitario, produciendo un espacio en el que se refuerza la reducción de la mujer al rol de madre-reproductora, dedicada al hogar, esposa/pareja.

La segunda modalidad de apropiación subjetiva encuentra en este intersticio que lo familiar habilita una posibilidad de vincularse con redes más amplias. En este caso, se trata de tomar como punto de partida ciertas formas o trazos de lo familiar, pero como una estrategia para hacer frente a la fragmentación y objetivación destituyente que recae sobre el territorio, para reforzar lo colectivo.

4 La noción de objetivación destituyente busca aportar a la comprensión de los procesos de subjetivación atravesados por la estigmatización. Tienen como característica el haber atravesado un proceso de destitución subjetiva, y se entienden como objetivaciones que operan en el borde de la subjetividad neoliberal, permitiendo una gestión de estos cuerpos juveniles marginalizados (Ghisigileri). 
A diferencia de la anterior, lo familiar aquí no remite a la familia nuclear, sino que está desplazada y/o superpuesta con diversos grupos de familias extensas, ensambladas e, incluso, no consanguíneas. Como señalaba Rosana: "Ellos viven con mucha naturalidad los lazos familiares que no son solo mamá y papá". Aunque sigue siendo muchas veces denominada en término de madre, aquí la madre puede ser la abuela, la tía, "la señora que lo/la cuidó" de niño o niña; así como la familia se vuelve extensiva a una amiga o una vecina. Las familias remiten a los lazos realmente existentes, en tanto se constituyen por las personas que efectivamente sostuvieron lugares de transmisión generacional y cuidado. Lo familiar como espacio de pertenencia, excede y se descentra de lo familiar entendido como nuclear/sanguíneo.

Las mujeres tienen también un lugar central en este entramado. Basándonos en los registros de observación, podemos aseverar que la participación de las reuniones barriales era exclusivamente femenina; así como eran mujeres quienes se encargaban de gestionar recursos, visitar a los hijos en las cárceles, acompañar en las actividades escolares.

En este sentido, esta apropiación subjetiva no queda exenta de la desigual distribución entre los géneros de las tareas de cuidado y la sobrecarga de las mujeres, pero a diferencia del anterior, está al servicio de la promoción y la agencia de otros modos de gestión de lo común. Esta situación se relaciona con una condición histórica de las mujeres que las convierte, como sostiene (Federici), en sujetas de lo común por excelencia.

$\mathrm{Al}$ respecto, Falú señala que las mujeres, a la par de que fueron sistemáticamente invisibilizadas desde el poder como sujetas de lo público, han estado íntimamente vinculadas con la construcción de sus ciudades, barrios, comunidades; y en especial, participando en los movimientos organizados en torno a demandas de mejoras habitacionales.

Esto condice con este caso de estudio, con la particularidad que aquí podemos notar también cómo, a partir de los procesos de destitución acaecidos, las últimas articulaciones comunitarias han tenido como participantes casi exclusivas a mujeres.

Yanina (Mujer adulta)- Porque le han dicho que es muy bueno el apoyo. La señora es mi prima. Y mi tía le ha comentado que está muy bueno porque mi otra prima trae los chicos acá. Y están adentro. No salen, no entra nadie que no sea de la casita, o sea, no permiten cosas raras.

Y bueno, fueron a hablar. Tenían miedo de venir, y eso que mi tía se crió acá en el barrio. Tenía miedo de traer a los hijos, pero ya que va ella, vemos. Iban a venir una semana, y si veían que la cosa iba mal, dejaban. Pero ya... siguen viniendo (risas). Acá se quedaron directamente (Entrevista, 13 de marzo de 2013).

Como se puede ver en este fragmento, una red familiar de mujeres -Yanina, la prima, la tía, otra primaproducen un canal que les permite acceder al servicio del apoyo escolar.

Sin embargo, a diferencia del familiarismo, su lógica no intercepta la comunitaria, sino que lo familiar se apoya en lo común y viceversa. Por ejemplo, en la situación recién analizada, el Proyecto Socio-educativo se vuelve investido por este recubrimiento familiar y de un adentro que resguarda de los desconocidosen contraposición al afuera en el que "pasan cosas raras"-.

A su vez, es de destacar que los vínculos familiares operan como una red capaz de sobreponerse al miedo que puede traspasar las fronteras que diferencian la villa del barrio, el adentrarse a una "zona 


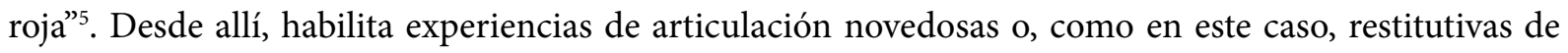
posibilidades vinculares.

Francisco- Yo lo que veo que tienen muy fuerte ustedes, son las relaciones, que tienen mucha red de ayuda entre ustedes, entre familiares y entre no familiares también. Que estaba mal... viniste a hablar con ella... Eloísa (Mujer adulta)- Sí, sí. Claro, acá tenemos... el otro día yo estaba bastante jodida y anduve hace pocos meses atrás enferma, y ella me decía: Vieja, dame el número de teléfono y yo te doy el mío para cualquier cosa, vos me llamás a mí y yo te ayudo. O yo la puedo ayudar a ella, lo mismo. Aunque ella siempre tiene alguien, yo no... estoy más sola.

Irma- Sí, yo a ella siempre la quise como familia, como si fuera una prima o una hermana, más que una amiga ¿viste? Yo le dije, estoy en las buenas y en las malas. Más ella, que vivió en mi casa... todo eso. Para mí, es una familia.

E- Claro, yo tengo el hermano de ella que para mí... Yo soy la vieja. Él se cruza de allá cuando no me ve y me dice: vieja, no te veo-dice. Acá estoy...

I- La hace reír un rato y después se va.

F- Tienen mucha historia compartida ustedes.

I- Nosotros siempre la quisimos a ella.

M- Siempre hemos sido, entre los padres, los hermanos, hemos sido una cosa, hemos compartido cosas buenas y malas, digamos. Cosas tristes... (Entrevista, 20 de mayo de 2017).

El acento está puesto en la productividad de esa vinculación que, si bien recurre a las formas de lo familiar, la excede. Lo familiar no como un sistema cerrado de relaciones, sino una frontera para otras vinculaciones posibles. Lo familiar y los vínculos comunitarios o extra-familiares, están atravesados por perímetros porosos que permiten el intercambio.

En esta línea, en las organizaciones comunitarias de Barranca Yaco era notoria la incidencia de las relaciones familiares. Las mismas se encontraban pobladas, o bien, por los/las familiares de la persona encargada de ese espacio - como el caso de la guardería/sala cuna-, o bien, por una articulación de un grupo de familias - como el caso del club.

Desde aquí, se pueda hacer otra lectura a las frecuentes críticas que registré -especialmente de agentes internos a la comunidad-, en las que esta situación era interpretada como un "acomodo", como un aprovecharse de lo común. Los argumentos que se argüían para sostener dicho punto de vista se basaban en que esa pertenencia garantizaba muchas veces el acceso a una beca o a cierta mercadería con destino comunitario (sean del Estado o de instituciones religiosas). En cambio, desde este análisis, se puede pensar también como una consecuencia lógica de las formas relacionales propias de esta comunidad, la cual encuentra en lo familiar un vínculo posible para gestionar sus recursos.

Esta pertenencia familiar -como posibilidad de "ser" otra identidad que la denostada- se juega en los y las jóvenes, o bien como posibilidad de constituir una familia nueva, o bien como una re-valorización de la familia de origen.

5 En el marco del nuevo Plan de seguridad ciudadana y prevención del delito, iniciado en 2016, la Ciudad de Córdoba fue clasificada a través de cuadrantes de vulnerabilidad social, siendo el cuadrante rojo el más vulnerable. Entre los pilares de esta política está la comprensión de que la inseguridad es inherente a la exclusión social (Crisafulli y Castro). 
Antes de ingresar al Proyecto, lo veo a Nicolás (17 años). Está sentado en una moto, sonríe y me saluda. Me acerco.

F- ¿Qué es de tu vida Nicolás? ¡Tanto tiempo!

$\mathrm{N}$ - Nada... nada bueno (sonríe con timidez).

Ante mis preguntas, me cuenta que no volvió a estudiar, y que tampoco trabaja.

N- Voy a ser papá.

F- ¡No me digás! ¿Quién es la mamá?

N- Una wacha de allá (Registro de campo, 24 de octubre de 2017).

En el caso de la constitución de nuevas familias, encontramos que las mismas se configuran desde precipitaciones de proyectos adolescentes o juveniles, y que pueden ser leídas desde este lugar como una forma de producir una pertenencia. En este caso, Nicolás contrapone lo "nada bueno" de su vida -el no estudiar, no trabajar- al "ser papá".

Rosario (12 años)- Hola, buenas tardes, estamos aquí con Lourdes y Martín. Le vamos a hacer unas preguntas.

Lourdes (14 años)- Hola.

Martín (16 años)- ¿Qué onda?

$\mathrm{R}-$ ¿Cuántos años tienen?

Lourdes - Yo tengo catorce

Martín - Yo dieciséis.

R- ¿Hace cuántos años están juntos?

Martín - Meses, diez meses.

$\mathrm{R}$ - ¿De dónde son?

Lourdes - De Córdoba Capital

Martín - De Córdoba Capital también, de mi casa.

R- ¿De qué barrio?

Lourdes - Pueyrredón

Martín - Alto General Paz

$\mathrm{R}-$ ¿Cómo se conocieron?

Lourdes- Éramos amigos del Facebook, y un día nos quisimos encontrar en la plaza del Híper, y bueno, nos encontramos.

Martín- Fue primero por Facebook, después por Whatsapp y de ahí de frente... usá la cabeza (Risas)

Lourdes - ¿Lo podemos hacer de nuevo?

Francisco- No, ¡está bien!

$\mathrm{R}$ - ¿Cómo se pusieron de novios?

Lourdes - Así.

Martín - Yo le pregunté si quería ser mi novia, obviamente.

R- ¿Cuándo y cómo se enteraron que iban a ser papás?

Lourdes - Nos enteramos a los cinco meses de embarazo que íbamos a ser papás, y yo le conté a él.

$\mathrm{R}$ - ¿Cómo se siente ahora?

Lourdes - Bien, felices.

Martín - Bien, por lo menos.

R- ¿Quieren tener otro bebé?

Lourdes - Por ahora no.

Martín - Por ahora no (se ríen). 
R- ¿Cómo se va a llamar?

Lourdes - Miguel Fermín

Martín - Oh, yo quería Pablo (risas)

R- ¿Qué va a ser? ¿Nena o varón?

Lourdes - Y... Si se llama Miguel, va a ser varón (Risas).

$\mathrm{R}-$ Bueno, gracias por responder nuestras preguntas y que la pasen lindo.

Lourdes - Chau (Entrevista en el marco de una radio autogestionada por niños, niñas y jóvenes del

Proyecto Socio-educativo - Registro de campo, 14 de noviembre de 2017).

En relación con las familias de origen, como hemos visto, la figura de la madre adquiere un lugar central, cuya relevancia se debe a que, muchas veces, son quienes sostienen posibilidades subjetivantes por fuera de los circuitos de estigmatización y destitución descritos.

Cuando salga de esta jaula,

con mi madre me encontraré...

Con un puchito en la mano,

una rosa y un clavel (fragmento de canción, Registro de campo, 21 de agosto de 2013).

En el anterior fragmento de la canción "Libertad" de Ulises Bueno ${ }^{6}$ se puede percibir cómo la madre emerge como una representante del afuera de la "jaula". Jaula que se puede en relación con el encierro de la cárcel, pero también de la exclusión, del aislamiento, de la objetivación destituyente. En este sentido, la madre se contrapone muchas veces a ciertas trayectorias de vida de jóvenes signadas por la falta de oportunidades.

Esta subjetivación que va de lo familiar a lo comunitario permite articular la siguiente observación, realizada en los talleres de jóvenes que tenía como actividad efectuar mapas barriales. A lo largo de los mismos pudimos apreciar cierta continuidad entre las jóvenes -mujeres- que a la hora de hacer sus cartografías resaltaron el lugar de los puentes como elemento central del territorio y quienes configuraron luego sus mapas barriales por casas-familias.

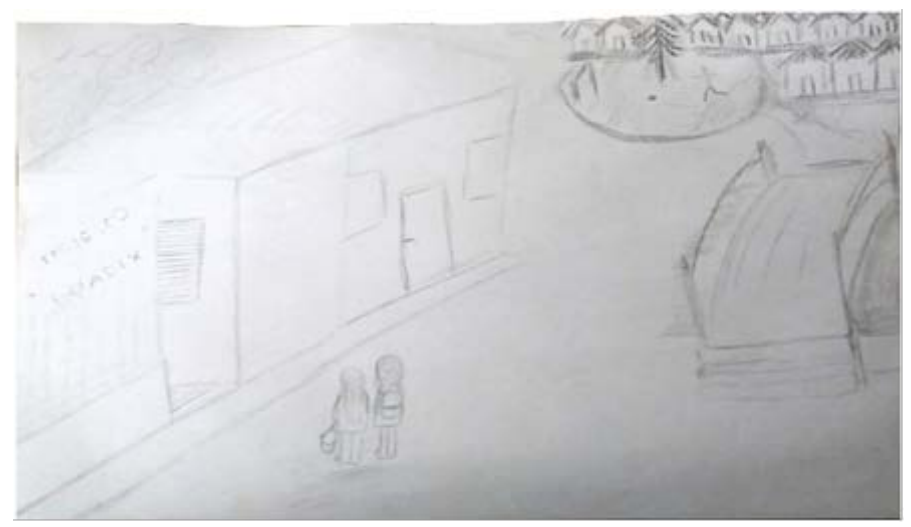

Figura 1. Dibujo realizado por una joven en el taller. Muestra a ella y su hermana, el proyecto Guadix, el puente "azul" y la "placita".

6 Cuyo registro tomé de un joven que lo cantaba en el Proyecto Socio-educativo. 
En sus dibujos - como se muestra en la figura 17-, luego de establecer el puente, empezaron a circunscribir las casas, y en cada una de ellas, los nombres de las familias que allí vivían: "acá vive la familia A", “acá la familia de B", etc. Desde esta modulación, la insistencia estaba en la "unión"; jerarquizando en el territorio los puentes por sobre las divisiones, y cuya articulación posible se daba a partir las relaciones por y desde las familias que se establecen en el barrio ${ }^{7}$.

Consideramos que aquí lo familiar está al servicio de la (posible) constitución de una trama vincular, de lo que podríamos definir, parafraseando a (Butler 17) un vínculo apasionado a la vinculación. Esta modulación subjetiva que se apoya en las posibilidades que brinda el estar con otro, les permite tejer "puentes" sobre la fragmentación reinante. Aquí lo familiar aparece como una forma de subjetivación posible, como un modo de ligazón y de sostén y, en última instancia, como un modo de resistencia.

\section{Problematizaciones al familismo}

Duschatzky y Corea, quienes también trabajaron con jóvenes de la Ciudad de Córdoba, coinciden en la relevancia que adquiere lo familiar en condiciones de pobreza como las estudiadas. Desde su argumentación, esto se debe a que emerge como un lugar de refugio y preservación. Relacionaron esta característica con los peligros y amenazas que representa el mundo extra-familiar en estos contextos. Es decir, frente a un entorno amenazante. Desde nuestra perspectiva, este argumento considera una dimensión importante que atraviesa los barrios en condiciones de pobreza, pero tiene sus limitaciones. Ubicar, como hacen estas autoras, a las amenazas como propio de lo extra-familiar y el resguardo como propio de lo intra-familiar, invisibiliza, en primer lugar, las múltiples violencias que se suceden en las familias (las cuales se agudizan al considerar la dimensión de género), así como las posibilidades de cuidado que ofrecen los lazos comunitarios e incluso el Estado, muchas veces más eficaces que los lazos familiares. En segundo lugar, no considera la porosidad y la inter-dependencia que existe entre lo comunitario y lo familiar, desde la cual esta división dicotómica resulta difícil de sostener: tanto las amenazas como los cuidados atraviesan y se retroalimentan entre ambos espacios.

En cambio, entendemos que, en gran medida, la producción de esta "comunidad familiar" se puede comprender desde una historia cuya textura está dada por la lucha entre, por un lado, las ya mencionadas estrategias de los sujetos para refugiarse y sostener su capacidad vincular y, por el otro, diversas estrategias del poder que, como veremos a continuación, permitieron dejar atrás una época caracterizada por una fuerte presencia de articulaciones comunitarias.

Podemos precisar que esta característica familista de Barranca Yaco se empieza a gestar a partir de los años 80, luego de un contexto de dictadura y autoritarismo. Antes de esto, hacia inicios de los años 70, encontramos una serie de actividades y movimientos comunitarios que dan cuenta de otro modo de

7 Esta actitud tiene cierto paralelismo con lo que pasa en la Ciudad. Mientras la racionalidad del Estado indica segregar y aislar, los y las jóvenes contraponen una acción colectiva como La Marcha de la Gorra. Es decir, también atraviesan la fragmentación espacial y construyen puentes. 
gestionar lo común ${ }^{8}$. En este período, se concentran en el barrio un grupo de personas de diferentes procedencias sociales. Los relatos nombran a una monja, profesionales de la salud, el cura "Gringo" Nelio Rougier, el "Gato", entre otros. Muchos de ellos viven en el barrio y realizan una tarea organizativa y de pedagogía sociopolítica, con una gran aceptación y colaboración de parte de las personas del lugar (Piotti, Relatos).

La represión que se inicia en Córdoba en 1974, y se consolida en el año 1976 con el último Golpe de Estado Cívico Militar en el contexto nacional, provoca la desarticulación de las diferentes experiencias de organización comunitaria y política que se habían gestado hasta entonces -muchas de ellas con los formatos de unidades básicas o asociaciones juveniles parroquiales-. Como señala Piotti (El terrorismo), imposibilitada la acción colectiva, el resistir se sostuvo en una actitud defensiva e implicó un repliegue a acciones individuales o familiares.

Es así que, desde los años 90, vemos lo que se denominó un retorno al familismo9', a una sobrevaloración de valores relacionados con lo familiar (Gundelach y Riis; Morá; Garzón). En el caso analizado, podemos asegurar que se relaciona con un proceso de destitución de los lazos comunitarios provocado por diversas estrategias de gobierno, que se inician (o refuerzan) con la represión llevada adelante por la Dictadura, y que luego se van reformulando. Estrategias que, por un lado, impulsaron sucesivos procesos de fragmentación social, segregación y un retraimiento de los espacios públicos, dejando a las ciudades latinoamericanas como lugares de desprotección y amenazas (Morá). Por el otro, persistieron en una valorización de lo familiar como espacio de pertenencia esencial y a las mujeres como sus principales protagonistas.

En este sentido es pertinente destacar que el feminismo latinoamericano fue una de las pocas corrientes que tempranamente, en los 80 , advirtió que la familia era "núcleo de valores del orden patriarcal10" (Kirkwood 11), al recluir a las mujeres a las funciones reproductivas, de cuidado y de socialización de los niños de manera exclusiva y esencial. Además, como tal, las familias eran también el lugar a dónde mirar para comenzar a desenredar el análisis de los autoritarismos.

Asimismo, las políticas públicas latinoamericanas se caracterizan por tener como soporte esta mirada familista (Sunkel). Los Estados (en cualquier régimen político) han hecho un uso estratégico de esta organización familiar a la hora de buscar la propagación de modelos, valores, prácticas (Sunkel). Estas políticas públicas apuntaron a consolidar una familia de tipo nuclear, con su división de roles tradicionales, dejando a las mujeres con las tareas de cuidado y, a su vez, como beneficiarias indirectas de la seguridad social, la cual se brindaba al hombre proveedor.

8 Como señala Gutiérrez, a diferencia de lo público, entendido como lo que es de todos y no es de ninguno: "Lo común, más bien, es lo poseído - o compartido- colectivamente por varios" (74).

9 La noción de familismo tiene como telón de fondo los estudios del antropólogo Banfield, quien definió el familismo amoral como la lealtad exclusiva a la familia, desestimando todo compromiso ético para con el resto de la sociedad, y contrapuesta a lo que llama cultura cívica, basada en la confianza interpersonal.

10 De acuerdo con Giberti, el patriarcado se concibe como: "un sistema político-histórico y social basado en la construcción de desigualdades que impone la interpretación de las diferencias anatómicas entre hombres y mujeres, construyendo jerarquías: la superioridad queda a cargo del género masculino y la inferioridad asociada al género femenino" (41). 
El régimen neoliberal que se consolida desde los años 90 agudiza incluso esta orientación de la política pública, en tanto transfiere a las familias muchas de las previsiones antiguamente a cargo de los Estados, e incorpora masivamente a las mujeres al mercado laboral, pero sin relevarlas de las responsabilidades de cuidado familiar (Sunkel 177).

Esto ha tenido un efecto duradero. En el siguiente fragmento del discurso del año 2005 del entonces Gobernador de la Provincia de Córdoba, José Manuel De la Sota ${ }^{11}$, da cuenta de ello. En el mismo, podemos ver cómo se articulan las condiciones descritas -familismo y políticas de hábitat, en este caso-, cuando se dirige a las 223 familias de Barranca Yaco que acordaron el traslado al desarrollo urbano que se hizo dentro del Programa de Hábitat Provincial12: "Nuevos Barrios: Mi Casa, Mi Vida" (Cervio, La Ciudad):

Quiero desearles que sean muy felices en estos nuevos hogares que a partir de hoy ustedes tienen. Quiero desearles que vean crecer los hijos sanos y fuertes. Que ustedes y sus esposos puedan conseguir el trabajo que falta, el empleo que no tienen. Quiero desearles a los abuelos que pasen los años que le quedan por vivir felices en compañía de sus hijos y de sus nietos (Gobierno de la Provincia de Córdoba)

Aquí se puede apreciar cómo la familia se nombra, se interviene, y se vuelve destinataria de políticas públicas. El formato de familia desde el que se interpela a los ciudadanos posee características claramente delimitadas: su espacio resulta ceñido al hogar, su función circunscrita al cuidado de los hijos y tienen como eje un vínculo de alianza entre esposos. Una estructura familiar con atribuciones de roles propios de una familia tradicional, nuclear y heteronormativa.

Desde los estudios de género y feminismos, como advertimos antes, las reflexiones críticas se centran en: la idealización de la familia y de la maternidad como "inclinación instintiva" y como mandato social que las recluye en los hogares (Villamizar); la constitución de un poder masculino sobre la generación y el género; la transferencia de múltiples funciones y responsabilidades estatales que deben ser gestionadas desde el hogar; y la consideración de que sólo la familia (nuclear, patriarcal, heterosexual y monogámica) puede resolver necesidades emocionales (Calveiro 266).

Todo esto redunda en una atribución y sobrecarga de roles domésticos en las mujeres, así como la inhibición a su acceso y agencia en el espacio público. Estas visiones desfavorecen las posibilidades de encontrar relaciones de solidaridad, de afecto o de ayuda mutua en otras instancias de la vida social entre las personas.

11 José Manuel De la Sota, perteneciente al Partido Justicialista, ha sido Gobernador de la Provincia de Córdoba en los períodos 1999-2007 y 2011-2015.

12 El Programa "Nuevos Barrios: Mi Casa, Mi Vida” fue decretado en el año 2002 por el Gobierno Provincial y contó con financiamiento del Banco Interamericano de Desarrollo (Peralta \& Liborio, 2014; Cervio, 2015); el mismo, mediante una importante intervención pública, aseguró la erradicación casi definitiva de los barrios humildes de las zonas centrales y peri-centrales de la Ciudad. Sobre las implicancias de este Programa existe abundante literatura. En el ámbito de las Ciencias Sociales de Córdoba, el programa de investigación Acción Colectiva (Scribano y Boito) y el colectivo de investigación Llano en Llamas (Núñez y Ciuffolini; Scarponetti y Ciuffolini) son referencias ineludibles. En sus análisis sostienen una visión crítica hacia el accionar del Estado y señalan las consecuencias negativas que tuvo esta política social para los sujetos afectados en términos de desconocimiento de sus necesidades, de aumento de la segregación socio-territorial y de complicidad con intereses de grandes grupos económicos, entre otros. 


\section{Reflexiones finales}

Desde estos desarrollos, podemos preguntarnos por las categorías que emergen del nombre del programa habitacional: "Nuevos Barrios: Mi Casa, Mi Vida", ya que los mismos adquieren una mayor densidad analítica. Estos "nuevos barrios" se perciben como nuevas configuraciones territoriales, nuevos regímenes de saber-poder que enlazan la "vida" a la "casa" y la casa a la familia.

Podemos pensar, desde aquí, en una tecnología de gobierno que se desplegó mediante políticas de solución habitacional con un fin estratégico: la producción de una nueva configuración social, un nuevo entramado relacional. Nuevos territorios en los que se busca reforzar el énfasis en lo privado, el hogar: reducir la vida a lo familiar. Estos "nuevos" y "mejorados" barrios conforman, en consecuencia, un sistema relacional constituido por núcleos de familias.

En este punto, son sugerentes los interrogantes que abre Villamizar referidos al familismo, ya que permiten poner de relive las condiciones que lo posibilitan: ¿Qué función estratégica lo vuelve viable? ¿Qué violencia requiere sostener invisbile esta idealización de lo familiar? ¿Qué le ofrece la sociedad a las familias y a las mujeres como contraprestación de semejante sobrecarga? (Villamizar 268)

A su vez, podemos decir que este repliegue familista se ubica en un intersticio del poder en el que los sujetos pueden llegar a refugiarse y construir articulaciones novedosas. Esta forma de subjetivación varía en tanto puede devenir, o bien, en una modalidad en la cual lo familiar se reduce a la pertenencia a determinado conjunto con asignaciones de roles de género tradicionales (patriarcales), y que eclipsa otras formas de entramados comunitarios; o bien, puede devenir en una forma de apropiación subjetiva en la que, si bien la desigualdad entre los géneros insiste, encuentra en los vínculos familiares una posibilidad y un soporte para procesos comunitarios más amplios.

Queda abierto el interrogante sobre la potencia que este modo de subjetivación tiene de constituirse en una resistencia a la fragmentación social, anteponiendo a lo fragmentario, una apuesta radical por hacer la vida con otros.

\section{Referencias}

Auyero, Javier y María Fernanda Berti. La violencia en los márgenes. Una maestra y un sociólogo en el conurbano bonaerense. Buenos Aires: Katz, 2013.

Banfield, Edwards. The moral basic of a backward society. Chicago: Free Press, 1958.

Bisig, Eleonor. Jóvenes y seguridad; control social y estrategias punitivas de exclusión. Código de Faltas Provincia de Córdoba. Córdoba: Universidad Nacional de Córdoba., 2014.

Butler, Judith. Los Mecanismos Psíquicos del Poder. Teorías Sobre la Sujeción. Madrid: Ediciones Cátedra, 2001.

Calveiro, Pilar. Familia y poder. Buenos Aires: Libros de la Araucaria, 2005.

Cardozo, Griselda. «Violencias y desigualdades: un análisis a partir de la experiencia de los y las jóvenes en Córdoba (Argentina).» Ponto-e-vírgula 17 (2015): 79-104.

Cervio, Ana. «Del "barrio social" a las "ciudades-barrios". Programas habitacionales y elaboración de sensibilidades en la ciudad de Córdoba (Argentina) durante las décadas de 1980 y 2000.» Methaodos. Revista de Ciencias Sociales 3.2 (2015): 175-191.

Cervio, Ana. «La ciudad como experiencia conflictiva: la problemática habitacional entre la gestión activa y la 
resistencia organizada». Scribano, Adrián. Mapeando interiores: cuerpo, conflicto y sensaciones. Córdoba: Jorge Sarmiento, 2007. 39-69.

Comisión Provincial de la Memoria de Córdoba. Mirar tras los muros. Situación de los derechos humanos de las personas privadas de libertad en Córdoba. Tercer informe de la Comisión de la Memoria. Córdoba, 2015.

Crisafulli, Lucas. «El martillo y la policía: hacia la tolerancia cero y el racismo.» Bisig, Eleonor. Jóvenes y seguridad; control social y estrategias punitivas de exclusión. Código de Faltas Provincia de Córdoba. Córdoba: Universidad Nacional de Córdoba, 2014. 177-198.

Crisafulli, Lucas y Julián Castro. «La metamorfosis de la Seguridad en Córdoba: Entre el actuarialismo y el delito de los pobres. .» I Jornadas de estudios sociales sobre delito, violencia y policía. La Plata: Universidad Nacional de La Plata. Facultad de Humanidades y Ciencias de la Educación., 20 y 21 de abril de 2017.

Di Leo, Pablo Francisco y Ana Clara Camarotti. «Relatos biográficos y procesos de individuación juveniles en barrios marginalizados de Argentina.» Revista Latinoamericana de Ciencias Sociales, Niñez y Juventud 2.15 (2017): 1021-1034.

Di Leo, Pablo y Roseni Pinheiro. «Vulnerabilidades y reconocimiento: abordaje biográfico de los vínculos entre jóvenes y violencias en barrios marginalizados de Buenos Aires, Argentina.» Cadernos de Saúde Pública 4.33 (2017): 1-11.

Duschatzky, Silvia y Cristina Corea. Chicos en banda. Los caminos de la subjetividad en el declive de las instituciones. Buenos Aires: Paidós, 2002.

Falú, Ana María. «El derecho de las mujeres a la ciudad. Espacios públicos sin discriminaciones y violencias. .» Revista Vivienda y Ciudad (2014): 10-28.

Federici, Silvia. Revolución en punto cero. Trabajo doméstico, reproducción y luchas feministas. Madrid : Traficantes de Sueños, 2013.

Foucault, Michel. Defender la sociedad. Curso en el College de France: 1975-1976. Buenos Aires: Fondo de Cultura Económica, 2000.

Foucault, Michel. «El sujeto y el poder.» Revista Mexicana de Sociología 3.50 (1988): 3-20.

Foucault, Michel. Historia de la Sexualidad 1. La voluntad del saber. Buenos Aires: Siglo XXI, 2008.

Foucault, Michel. La Arqueología del Saber. Buenos Aires: Siglo Veintiuno Editores, 2002.

Foucault, Michel. Nacimiento de la biopolítica: Curso en el Collège de France: 1978-1979. Buenos Aires: Fondo de Cultura Económica, 2007.

Foucault, Michel. Seguridad, territorio y población: Curso en el College de France: 1977-1978. Buenos Aires: Fondo de Cultura Económica, 2006.

Foucault, Michel. «Verdade e subjectividade.» Revista de Comunicação e linguagem 19 (1993): 203-223.

Foucault, Michel. Vigilar y Castigar: nacimiento de la prisión. Buenos Aires: Siglo Veintiuno Editores, 2008.

Garzón, Adela. «Familismo y creencias políticas.» Psicología Política 17 (1998): 101-128.

Ghisigileri, Francisco. Procesos de subjetivación de jóvenes en condiciones de pobreza de un barrio de la Ciudad de Córdoba. Tesis doctoral. Córdoba: Universidad Nacional de Córdoba, 2020.

Giberti, Eva. La familia, a pesar de todo. Buenos Aires: Noveduc, 2005.

Gobierno de la Provincia de Córdoba . «De la Sota entregó 223 viviendas en Barrio Renacimiento. .» 4 de marzo de 2005. <Recuperado de http://web2.cba.gov.ar/web/audiopress.nsf/0fb4dfb09eb2b61603256970005f59cd/2048a 3105cce53f203256fba00776520? OpenDocument>.

Gundelach, Peter y Ole Riis. «¿El retorno al familismo?» Díez, Nicolás y Ronald Inglehart. Tendencias mundiales de cambio en los valores sociales y políticos. Fundesco, 1994.

Gutiérrez, Raquel. Horizontes comunitarios-populares. Producción de lo común más allá de las políticas Estadocéntricas. Madrid: Traficantes de Sueños, 2017.

Kirkwood, Julieta. El feminismo como negación del autoritarimos. Santiago de Chile: Flacso: Flacso, 1983.

Marengo, Cecilia y Ana Elorza. «Globalización y Políticas Urbanas. La política habitacional focalizada como estrategia para atenuar condiciones de pobreza urbana: los programas implementados en Córdoba y los desafíos pendientes.» Cuaderno Urbano. Espacio, Cultura, Sociedad 8 (Marengo, C.; Elorza, A. L.): 7-33.

Míguez, Daniel. Los pibes chorros: estigma y marginación. Buenos Aires: Capital Intelectual, 2010.

Morás, Luis Eduardo. «Nacer siendo menor. Reflexiones sobre punitividad, crisis institucional y adolescentes en conflicto con la ley» (Coord.), Miguel Serna. Pobreza y (des)igualdad en Uruguay: una relación en debate. Montevideo: UR. FCS-DS: ASDI: Clacso, 2010. 267-284. 
Nateras, Alfredo. "Geografías y mapas de las juventudes contemporáneas en México y América Latina.» Revista Somepso 3.2 (2018): 54-73.

Nateras, Alfredo. «Juventudes Sitiadas y Resistencias Afectivas.» México: Gedisa, 2016. 59-22.

Núñez, Ana y María Alejandra Ciuffolini. Política y territorialidad en tres ciudades argentinas. Buenos Aires: El Colectivo, 2011.

Piotti, María Lidia. «El terrorismo de Estado y la militancia territorial en la Ciudad de Córdoba.» Encuentro: Memorias y reflexiones a 40 años del Golpe de Estado de 1976. Córdoba: Universidad Nacional de Córdoba, 2016.

Piotti, María Lidia, Elorza, Ana Laura y Morillo, Ernesto. «Relatos, desde las memorias de sus protagonistas, sobre la acción colectiva en Villas de Córdoba durante el período 65- 76.» Encuentro Internacional: Fecundidad de la memoria. Desafíos del presente a los usos del pasado en América Latina. Córdoba: Centro de Estudios Avanzados de la Universidad Nacional de Córdoba, 2011.

Reguillo, Rossana. «Las múltiples fronteras de la violencia: jóvenes latinoamericanos entre la precarización y el desencanto.» Pensamiento Iberoamericano 3 (2008): 205-225.

Scarponetti, Patricia y Alejandra Ciuffolini. Ojos que no ven, corazón que no siente. Relocalización Territorial y conflictividad social: un estudio sobre los barrios ciudades de Córdoba. Buenos Aires: Nobuko, 2011.

Scribano, Adrián y Eugenia Boito. El purgatorio que no fue: Acciones profanas entre la esperanza y la soportabilidad. Buenos Aires: Ciccus, 2010.

Scribano, Adrián y María Eugenia Boito. El purgatorio que no fue: Acciones profanas entre la esperanza y la soportabilidad. Buenos Aires: Ciccus, 2010.

Sunkel, Guillermo. « Regímenes de bienestar y políticas de familia en América Latina. .» Arriagad, Irma. Gestión y financiamiento de las políticas que afectan a las familias. Santiago: Cepal, 2007. 167-176.Villamizar, Yolanda. «Las familias y las relaciones de género. El familismo: una crítica desde la perspectiva de género.» Arévalo Naranjo, Liz, et al. Familias, Cambios y Estrategias. Violencia contra las mujeres/Violencia de género. Colombia: Centro de Estudios Sociales, 2005. 263-277. 